\title{
Structure of magnetic domain wall in cylindrical microwire
}

\author{
Andrzej Janutka* and Przemysław Gawroński ${ }^{\dagger}$ \\ *Institute of Physics, Wroclaw University of Technology, 50-370 Wrocław, Poland \\ ${ }^{\dagger}$ Faculty of Physics and Applied Computer Science, AGH University of Science and Technology, 30-059 Krakow, \\ Poland
}

\begin{abstract}
Within a simple model, we study magnetic domain walls (DWs) inside the inner core of the amorphous ferromagnetic microwire whose spin ordering is a core-shell structure. The interaction of the (internal-stress created) outer shell of the wire on the inner core is included into the Landau-Lifshitz equation via an effective Dzyaloshinskii-Moriya-like anisotropy. Resulting DW textures are classified. The model is applicable to the nanowires of a modulated diameter (periodically constricted nanowires) as well. In that case a core-shell magnetic structure is of the purely-magnetostatic origin. Because the micromagnetic simulations of the microwires are extremely challenging, the simulations of the structured nanowires are performed with the purpose of verifying analytical predictions on the shape of the DW.
\end{abstract}

Index Terms-Magnetic domain walls, magnetic wires.

\section{INTRODUCTION}

Magnetic amorphous glass-coated wires of the micron-sized diameter are very important objects for sensor manufacturing while the process of the wire remagnetization [via the domainwall (DW) propagation] is a basics of the sensing mechanism (next to the giant magneto-impedance; GMI effect). However, the sufficiently detailed while simple model of the DW statics and dynamics that would enable the description of the microwire remagnetization is still missing. Such a model should map a complex core-shell ordering of the microwire. The inner core is composed of large ferromagnetic domains which are magnetized parallel to the wire axis due to the dipole-induced shape anisotropy of the easy-axis type. In an external field these domains shrink or expand driving the DW propagation. Unlike the outer-shell observations, the experimental studies of the inner-core magnetization are indirect, thus, they require a model-based interpretation. The minimal model for describing the remagnetization should include the influence of a complex ordering of the outer shell (a bamboo-like patterned structure) on the inner-core DW. It would enable estimations of dynamical characteristics with dependence on the parameters of the DW shape. An effective (analytical) approach is preferable since, for typical (e.g. Fe-Si-B, Co-Si-B based) amorphous microwires, the presence of a strong internal stress in the wire makes the simulations complex [1], [2]. More importantly, the (long-range) dipole (magnetostatic) spin interactions interactions cannot be efficiently included into the numerical treatment since systems to consider are huge compared to the

E-mail: Andrzej.Janutka@pwr.wroc.pl magnetostatic exchange length of the materials (a parameter that limits the sizes of the wire discretization cell). Note that the magnetostatics has been established to influence the DW-assisted remagnetization of the amorphous glass-coated microwires as well as the volume remagnetization of short (non-bistable) GMI microwires [3], [4], [5].

The aim of the present paper is to provide the relevant model of the DW. The description of the inner core of the softferromagnetic wire is formulated within the Landau-LifshitzGilbert (LLG) equation in 3D that we solve analytically. In the glass-coated amorphous or nanocrystalline ferromagnetic wires, the magnetic ordering of the outer shell is due to a radially-distributed internal stress [6], [7], [8]. The stress is induced during the manufacturing process that contains the rapid solidification and slow cooling stages [9], [10], [11]. The distribution of the stress can be then modified removing the glass coating or applying a thermal treatment [12], [13], [14], [15]. Since the outer-shell pattern can be very complex and dependent on small fluctuations of the parameters of the manufacturing process, we include the effect of that pattern on the inner core "fenomenologically", (with a single numerical parameter only), via a Dzyaloshinskii-Moriya-like (DM) contribution to the LLG equation.

A similar to the above described core-shell magnetic ordering of a ferromagnetic wire can be induced without the internal stress while via a modulation of the wire diameter, thus, the modulation of the magnetic-charge distribution on the wire surface [16]. The present model of the inner-core ordering is applicable to such structured wires, which enables us to compare the analytical DW solutions to the model with the micromagnetically simulated DWs in the periodically constricted nanowires.

Upon formulating the fundamentals of the model in Sec. II, in Sec. III, we present details of single-DW solutions. For a comparison, we perform micromagnetic simulations of the DWs in periodically constricted nanowires. In Sec. IV, we discuss aspects of the field-driven propagation of the DW which are connected to the model. Conclusions are collected in Sec. V.

\section{MOdEL}

Describing the inner core of a ferromagnetic wire, we consider the LLG equation in $3 \mathrm{D}$ in the form

$$
-\frac{\partial \mathbf{m}}{\partial t}=\frac{J}{M} \mathbf{m} \times \Delta \mathbf{m}+\frac{\beta}{M}(\mathbf{m} \cdot \hat{i}) \mathbf{m} \times \hat{i}+\gamma \mathbf{m} \times \mathbf{H}
$$




$$
-\frac{d}{M} \mathbf{m} \times\left(\hat{i} \times \frac{\partial \mathbf{m}}{\partial x}\right)-\frac{\alpha}{M} \mathbf{m} \times \frac{\partial \mathbf{m}}{\partial t} .
$$

Here, $\hat{i} \equiv(1,0,0)$, (the wire is directed along the $x$ axis), $M=|\mathbf{m}|, \gamma$ denotes the gyromagnetic factor, $J$ denotes the exchange constant, $\mathbf{H}=\left(H_{x}, 0,0\right)$ represents the external (longitudinal) magnetic field. The parameters $\beta$ and $d$ determine the strength of the effective easy-axis anisotropy (of the magnetostatic origin, $\beta \leq \gamma \mu_{0} M^{2}$ ) and a DMlike anisotropy, respectively. The DW solutions satisfy the condition $\lim _{|x| \rightarrow \infty} \mathbf{m}= \pm(M, 0,0)$.

The effective anisotropy of the DM type matches the properties of the dependence on the magnetization derivative over the wire-axis coordinate (a consequence of the shell patterning) and of the non-invariance with respect to the space inversion. The non-invariance results from the chirality of the ordering inside the outer shell.

In the periodically constricted wire, the DM-like anisotropy is due to a modulation of the projection of the wire-surface normal onto the long axis of the system. The inhomogeneous (periodic) distribution of the surface magnetic charges induce a modulation of the outer-shell ordering, thus, modifying the axial distribution of the anisotropy in the inner core. In the amorphous glass-coated microwire, the origin of the outershell ordering is different while the result of the mutual core and shell interaction is a similar helical ordering of the overall system [16], [17]. The outer-shell magnetization of the glasscoated amorphous microwire is circumferential in the case of the negative-magnetostriction materials while radial in the case of the positive magnetostriction. Additionally, the shell is divided into micro-domains because of the magnetostatics.

Looking for the solution to [1], following [18], we apply the transform

$$
m_{+}=\frac{2 M}{f^{*} / g+g^{*} / f}, \quad m_{x}=M \frac{f^{*} / g-g^{*} / f}{f^{*} / g+g^{*} / f},
$$

where $m_{ \pm} \equiv m_{y} \pm \mathrm{i} m_{z}$. Thus, we introduce secondary dynamical parameters $g(x, y, z, t), f(x, y, z, t)$ which take complex values and we ensure that the constraint $|\mathbf{m}|=M$ is satisfied. The purpose is to obtain the secondary equations of (unconstrained) motion in a tri-linear form, namely

$$
\begin{array}{r}
-f \mathrm{i} D_{t} f^{*} \cdot g=f\left[\alpha D_{t}+J\left(D_{x}^{2}+D_{y}^{2}+D_{z}^{2}\right)\right. \\
\left.+\mathrm{i} d D_{x}\right] f^{*} \cdot g+J g^{*}\left(D_{x}^{2}+D_{y}^{2}+D_{z}^{2}\right) g \cdot g \\
-\left(\gamma H_{x}+\beta\right)|f|^{2} g \\
-g^{*} \mathrm{i} D_{t} f^{*} \cdot g=g^{*}\left[\alpha D_{t}-J\left(D_{x}^{2}+D_{y}^{2}+D_{z}^{2}\right)\right. \\
\left.-\mathrm{i} d D_{x}\right] f^{*} \cdot g-J f\left(D_{x}^{2}+D_{y}^{2}+D_{z}^{2}\right) f^{*} \cdot f^{*} \\
+\left(-\gamma H_{x}+\beta\right)|g|^{2} f^{*} .
\end{array}
$$

Here $D_{t}, D_{x}, D_{y}, D_{z}$ denote Hirota operators of differentiation $D_{x}^{n} b(x, y, z, t) \cdot c(x, y, z, t) \equiv(\partial / \partial x-$ $\left.\partial / \partial x^{\prime}\right)\left.^{n} b(x, y, z, t) c\left(x^{\prime}, y^{\prime}, z^{\prime}, t^{\prime}\right)\right|_{x=x^{\prime}, y=y^{\prime}, z=z^{\prime}, t=t^{\prime}}$.

Specific solutions of the multi-linear partial differential equations in the form of expansions over trial (exponential) functions correspond to multi-soliton solutions to primary nonlinear equations. This fact enables a systematic study of multi-DW solutions to the LLG equation. In terms of the single-DW solutions, using (3) instead of (1) is convenient when verifying the results of the next section.

\section{DOMAIN-WALl STRUCTURE}

For the case $H_{x}=0$, Single-DW solution to (3) has been found in the form

$$
f=1, \quad g=u \mathrm{e}^{k x+q y+p z},
$$

where

$$
k^{2}+q^{2}+p^{2}-\frac{\mathrm{i} d k}{J}=\frac{\beta}{J}
$$

and $\operatorname{Re} k \neq 0$. We denote $k \equiv k^{\prime}+\mathrm{i} k^{\prime \prime}, q \equiv q^{\prime}+\mathrm{i} q^{\prime \prime}, p \equiv$ $p^{\prime}+\mathrm{i} p^{\prime \prime}$, where $k^{\left(^{\prime \prime}\right)}, q^{\prime\left({ }^{\prime \prime}\right)}, p^{\prime\left({ }^{\prime \prime}\right)}$ take real values, rewriting (5) with

$$
\begin{array}{r}
k^{\prime 2}+q^{\prime 2}+p^{\prime 2}-k^{\prime \prime 2}-q^{\prime \prime 2}-p^{\prime \prime 2}=\frac{\beta}{J}-\frac{d k^{\prime \prime}}{J}, \\
k^{\prime} k^{\prime \prime}+q^{\prime} q^{\prime \prime}+p^{\prime} p^{\prime \prime}-\frac{d k^{\prime}}{2 J}=0 .
\end{array}
$$

Assuming the DW to be centered at $(x, y, z)=0$, (then $u=\mathrm{e}^{\mathrm{i} \phi}$ ), the relevant magnetization profile [the single-DW solution to [1]] is written explicitly with

$$
\begin{aligned}
& m_{+}(x, y, z)=M \mathrm{e}^{\mathrm{i}\left(\phi+k^{\prime \prime} x+q^{\prime \prime} y+p^{\prime \prime} z\right)} \operatorname{sech}\left[k^{\prime} x+q^{\prime} y+p^{\prime} z\right] \\
& m_{x}(x, y, z)=-M \tanh \left[k^{\prime} x+q^{\prime} y+p^{\prime} z\right] .
\end{aligned}
$$

For $q=p=0$, the twisted transverse DW solution is obtained unless

$$
\begin{array}{r}
k^{\prime \prime}=\frac{d}{2 J}, \\
k^{\prime 2}=\frac{\beta}{J}-\frac{d^{2}}{4 J^{2}} .
\end{array}
$$

For $q^{\prime}=p^{\prime}=0$ and $\left|k^{\prime \prime}\right| \ll\left|q^{\prime \prime}\right|=\left|p^{\prime \prime}\right|,\left|k^{\prime}\right|$, the vortex DW of Fig. 1a is found unless the condition (8) and

$$
k^{\prime 2}-2 q^{\prime \prime 2}=\frac{\beta}{J}-\frac{d^{2}}{4 J^{2}}
$$

are satisfied. The solution of the type of the twisted transverse DW is expected not to be relevant to thick wires since it corresponds to a magnetization that is constant within the cross sections of the inner core.

Without loose of generality, we assume $p^{\prime}=0$, thus, we choose a DW orientation in the YZ plane. The (dipoleinduced) shape anisotropy that points the inner-core magnetization parallel to the wire axis, drives the core-shell interface to be a local easy plane of the magnetization. We utilize this notice in order to reduce the number of free parameters. Namely, on the contour of the intersection of the DW-plane $\left(k^{\prime} x+q^{\prime} y=0\right)$ with the core-shell interface $\left(\sqrt{y^{2}+z^{2}}=R\right)$, the magnetization should be aligned in the circumferential direction wherever possible, in particular, at the central $(x=0)$ and boundary ( $\left.x= \pm q^{\prime} R / k^{\prime}\right)$ cross-sections of the wire in the DW-plane area. Therefore, $q^{\prime \prime}=0,|\phi|=\pi / 2,\left|p^{\prime \prime}\right| R=\pi / 2$. Finally, from (6), one finds (8) and

$$
k^{\prime 2}+q^{\prime 2}=\beta / J-d^{2} / 4 J^{2}+(\pi / 2 R)^{2} .
$$

to be satisfied. An additional "freedom" on the solution is due to independence of (1) of the first derivatives of $\mathbf{m}$ over $y$ and 
a)
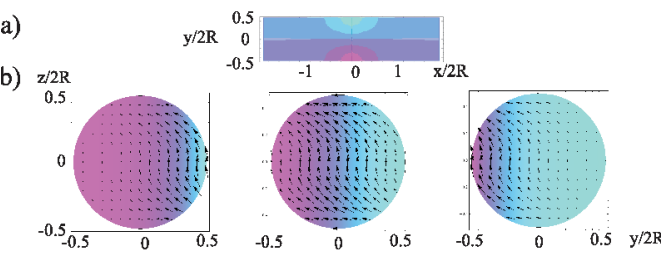

$y / 2 R \quad 0.5$
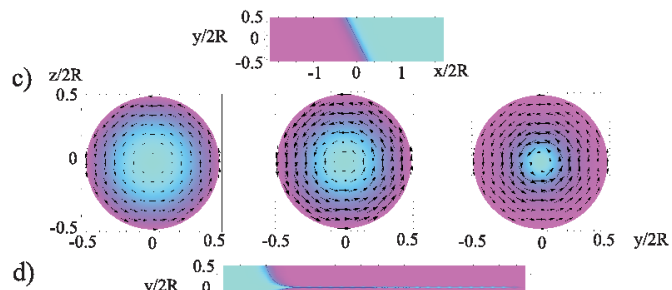

d)
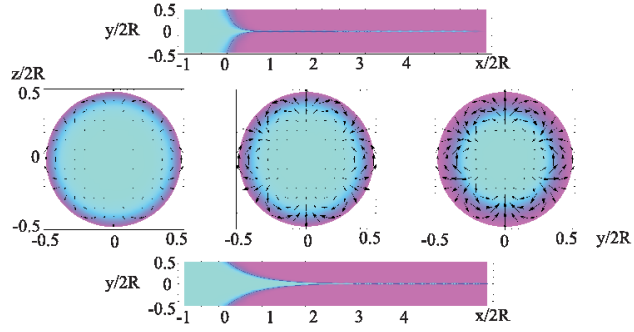

Fig. 1. The magnetization in the cross-sections of the inner core in the vortex DW at $z=0$ (a), in the planar-DW at $x=-q^{\prime} R / k^{\prime}, x=0$, $x=q^{\prime} R / k^{\prime}$, from left to right, and at $z=0(\mathrm{~b})$, as well as the conical-DW at $x=-1 /\left|k^{\prime}\right|, x=0, x=1 /\left|k^{\prime}\right|$, from left to right, and at $z=0$, for $\eta=1$ (c), and $\eta=4$ (d). For a)-d); $\left|k^{\prime \prime}\right| \ll\left|k^{\prime}\right|=\pi / R, q^{\prime} / k^{\prime}=1 / 2$. In a), the colors (red or blue) and its intensity indicate the sign and value of the $\mathrm{z}$ component of the magnetization, whereas in b)-d), the sign and value of the $x$ component of the magnetization. The arrows indicate the projection of the magnetization onto the $\mathrm{YZ}$ plane.

$z$ coordinates, which allows the perpendicular magnetization to take the form

$$
m_{+}(x, y, z)=M \mathrm{e}^{\mathrm{i}\left(\phi+k^{\prime \prime} x+p^{\prime \prime}|z|\right)} \operatorname{sech}\left[k^{\prime} x+q^{\prime} y\right]
$$

instead of (7). The relevant DW texture shown in Fig. 1b corresponds to a "planar" DW of [19]. A similar DW albeit deformed at its ends with a small flexture of the DW plane has been found with the micromagnetic simulations for the constricted nanowires [16], [20].

Using a different from (4) ansatz

$$
f=1, \quad g=\mathrm{e}^{k x+\mathrm{i} \phi+\mathrm{i} \eta \cdot \arctan (z / y)}\left(\frac{\sqrt{y^{2}+z^{2}}}{\mathcal{R}}\right)^{|\eta|}
$$

whose map onto the YZ plane represents the (BelavinPolyakov) Skyrmion [21], we find it to satisfy (3) under the conditions (8)- 9 . We expect $\mathcal{R}$ to be close to $R$. The relevant magnetization field is plotted in Figs. 1c, 1d, where the DW is seen to be asymmetric relative to the YZ plane. The present structure is singular at $(x, y, z) \rightarrow(\infty, 0,0)$ (the DW is infinitely long on the central line of the wire) and corresponds to a "tubular-conical" DW observed in [22], [23]. We expect the ordering in the DW to resemble the ordering in

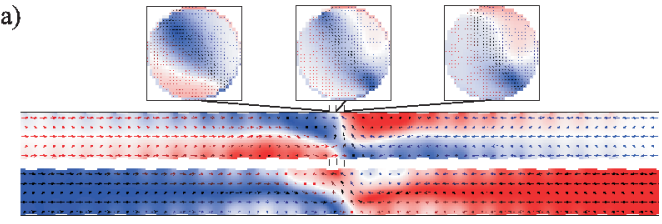

b)

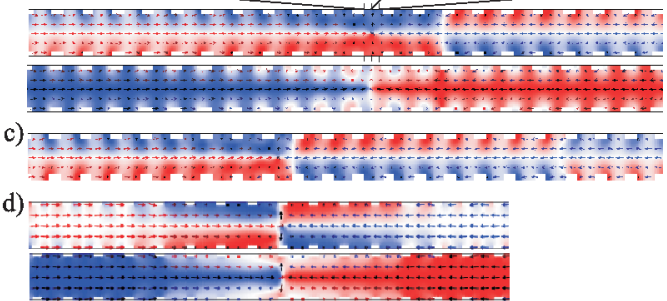

Fig. 2. The magnetization in the longitudinal $(z=0)$ and in the transverse ( $x=$ const) cross-sections of the constricted Py nanowire in the DW area obtained with the micromagnetic simulations. The outer diameter of the wire is $D=150 \mathrm{~nm}$, the constriction diameter is: $0.9 D$ (a), $0.6 D$ (b), $0.5 D$ (c), 0.7D (d). Stable DWs of the planar type (a), vortex type (b), (c), and metastable (conical-like) DW (d) are shown indicating the sign and value of the $\mathrm{z}$ (x) component of the magnetization at the top (bottom) pictures with the colors (red to blue).

the outer shell. Hence, the solution with $\eta=1$ is expected to be valid to the glass-coated negative-magnetostriction wires, (in particular, Co-rich microwires), whose shell is circumferentially magnetized. The solutions with higher values of $|\eta|$ are expected to describe the DWs in the wires of positivemagnetostriction materials, (in particular, Fe-rich microwires). Then $2(|\eta|-1)$ is equal to the number of the radiallymagnetized microdomains in each segment of the bamboostructured outer shell. Obviously, the conical DW cannot be a stable solution to the LLG equation. The elongated vertex of such a DW has to shrink with time in order to reduce the exchange energy. The instability of very elongated DWs in amorphous glass-coated microwires has been observed [24]. A reported strong dependence of the width of such DWs on the longitudinal external field coincides with the sensitivity of the outer-shell ordering to the field and suggests the $\eta$ parameter of the DW to depend on the field intensity [25]. In the external field, the outer shell of the negative-magnetostriction microwire can be of a complex vortex-containing texture, thus, we anticipate DWs of $|\eta|>1$ to be preferable in it [26].

In order to verify the validity of our effective approach in terms of predicting the DW structure in core-shell ordered wires, we have performed micromagnetic simulations of the DWs in constricted soft-magnetic nanowires. We have considered Py nanowires (the saturation magnetization $M=$ $8.6 \cdot 10^{5} \mathrm{~A} / \mathrm{m}$, the exchange stiffness $A_{e x}=1.3 \cdot 10^{-11} \mathrm{~J} / \mathrm{m}$ ) since Py is the most basic soft-magnetic material for the nanoscale applications. This makes our simulations realistic, while typical Metglas materials of the microwires are difficult 
to process to the nanoscale without inducing large internal stress. Simulating infinitely-long nanowires, unlike [16], we have discretized very $(9 \mu \mathrm{m})$ long systems. The discretization size was $5 \mathrm{~nm}$, (we utilized the OOMMF package), while the simulations were initialized with a stepwise magnetization distribution, (an infinitely narrow DW). The central $3 \mu \mathrm{m}-$ long fragment of the wire has been pre-calculated. Fixing the magnetization at the ends of the wire mesh, we counter the tendency towards reducing the surface magnetic charge. If we did not so, it would block the expansion of the bamboostructure of the outer shell along the wire. We have kept constant the outer diameter $D=150 \mathrm{~nm}$ and the length of the constrictions while we have varied the diameter of the constrictions. Thus, we have controlled the strength of a helical anisotropy with a single parameter.

According to Fig. 2, the radius of the axially-magnetized area of the nanowire is found to be significantly smaller than the constriction radius, thus, the ordering is of a genuine coreshell type. The outer shell of the wire is seen to form a bamboo-like domain structure whose period decreases with decreasing the constriction diameter. The stable DWs are found to be of the planar type (Fig. 2a) or of the vortex type (Figs. 2b, 2c) for the constriction diameter bigger or smaller than about $0.8 \mathrm{D}$, respectively. It is in a correspondence with our model that predicts the planar DWs to be formed provided $\beta>d^{2} / J$ because of 11 ), (a weak modulation of the surface layer of the wire), while the vortex-DW formation to allow the opposite relation $\beta<d^{2} / J$. The relaxation to the vortex DW structure as well as to the planar DW structure follows the formation of a meta-stable conical DW (Fig. 2d). In the simulations, we do not observe other DWs than predicted with our analytical model. Hence, we claim the model that combines the uniform easy-axis and a DM-like anisotropy to be capable to effectively describe the DWs of the core-shell ordered system.

Let us notice that the vortex DWs have been previously found in the circular-cross-section nanowires of the magnetocrystalline easy-plane anisotropy [27], while the planar and meta-stable conical DWs have been predicted to result from the competition between the magnetostatics and complex exchange interactions [20], [28]. Our DW simulations enhance the motivation for describing both types of the DWs within a single model however.

With regard to the amorphous microwires, a simple estimation of $d$ is based on the disturbance of the stress-induced magnetic field in the wire $H \sim 3|\lambda| \sigma / \mu_{0} M$, where $\lambda$ denotes the magnetoelastic constant. Here, the stress $\sigma=\sigma_{i}+\sigma_{a}$ consists of the internal and applied contributions. The internal stress is a complicated function of the parameters of the wire composition and fabrication conditions (the initial temperatures of the wire and of the glass cover, an extraction stress) [10]. The first differential correction to this stress due to the ordering inhomogeneity (a magnetostatically-induced micro-domain structure) is expected to be of the order of $\sigma l_{m s}|\nabla \times \mathbf{m}| / M$, where $l_{m s}=\left(2 A_{e x} / \mu_{0} M^{2}\right)^{1 / 2}$ denotes the magnetostatic exchange length, $\left(A_{e x}=J M / 2 \gamma\right)$. This leads to the estimate $d \sim \gamma 3 / 2 \cdot|\lambda| \sigma l_{m s} \equiv \gamma K_{m e} l_{m s}$.

\section{IMPLICATIONS FOR DOMAIN-WALL DYNAMICS}

Let us denote any stationary single-DW solution to (3) by $f_{0}(x, y, z), g_{0}(x, y, z)$. The inclusion of $H_{x} \neq 0$ leads to the dynamical solutions $f(x, y, z, t)=f_{0}(x, y, z)=1$, $g(x, y, z, t)=\mathrm{e}^{\mathrm{i} \omega t} g_{0}(x, y, x)$, where $\omega \equiv \omega^{\prime}+\mathrm{i} \omega^{\prime \prime}=$ $-\gamma H_{x} /(1+\mathrm{i} \alpha)$. It describes the driven motion of the DW with the velocity $v=\omega^{\prime \prime} / k^{\prime} \equiv S H_{x}$ accompanied by the magnetization rotation about the wire axis with the frequency $\omega^{\prime}$. Here, $S \approx \gamma \alpha /\left|k^{\prime}\right|$ denotes the DW mobility. However, the Walker breakdown is observed in the positive-magnetostriction amorphous and nanocrystalline microwires [29]. In the regime of the viscous motion of the DW $\left(\omega^{\prime}=0\right)$, taking the 1.h.s. of (1) and (3) equal to zero, one finds $S=\gamma / \alpha\left|k^{\prime}\right|$. Since we consider the magnetization of the outer shell of the wire to be stationary in the YZ plane, easy directions in the cross-sections of the inner core. Thus, the DW motion can be viscous [30]. For instance, the required discrete rotational symmetry of the shell is relevant to the conical DW of the parameter $|\eta|>1$.

The measurements of the DW mobility for the glass-coated microwires with dependence on the applied stress has been performed in [31]. In the cited paper, a qualitative analysis for the viscous regime of the DW motion has been performed taking $S=\gamma l_{m e} / \alpha$. There, the width of the DW has been assumed to be the magnetoelastic exchange length $l_{m e}=\left(2 A_{e x} / K_{m e}\right)^{1 / 2}$. For sufficiently high stress applied, when $k^{\prime 2} \sim d^{2} / 4 J^{2} \gg \beta / J$, (the vortex-DW regime), the above assumption corresponds to taking $d \sim \gamma K_{m e} l_{m e}$ instead of the previous-section estimate $d \sim \gamma K_{m e} l_{m s}$. It is a consequence of neglecting the dipole-induced anisotropy while assuming the anisotropy in the outer shell of the wire to be of the purely magneto-elastic origin. In the consequence, the cited authors find $S \propto 1 / \sqrt{\sigma}$, (in a disagreement with experimental data), while we evaluate $S=\gamma l_{m e}^{2} / l_{m s} \alpha \propto 1 / \sigma$. However, the magnetostatic origin of the domains inside the outer-shell has been established previously for the Fe-rich microwires [3], and for Co-rich microwires and nanowires [4]. Moreover, following [11], the existence of the outer shell cannot be explained with the exchange and magnetoelastic spin interactions only, while the distribution of the magnetic charges must be taken into account [25]. A simple comparison of typical magnetoelastic and magnetostatic exchange lengths of the amorphous microwires $\left(K_{m e} \sim 10^{4} \mathrm{~J} / \mathrm{m}^{3}\right.$ for $\mathrm{Fe}-\mathrm{Si}-\mathrm{B}$, $K_{m e} \sim-10^{3} \mathrm{~J} / \mathrm{m}^{3}$ for $\mathrm{Co}-\mathrm{Si}-\mathrm{B}$, and $\mu_{0} M^{2} \sim 10^{5} \mathrm{~J} / \mathrm{m}^{3}$ ) shows $l_{m s}^{2} / l_{m e}^{2} \leq 1 / 10$ [1], [32]. Since the smallest characteristic exchange length is expected to determine the thickness of the outer-shell DWs, following the previous section, we claim the parameter $d$, thus, the mobility of the inner-core DW to be dependent on the magnetostatic exchange length $l_{m s}$.

Despite the (Hirota) method that we apply is capable to treat the two-soliton states, the description of the DW collision is complex because of the necessity of including the dissipation. However, the result of the collision can be predicted on the basis of a simple rule that has been verified for ferromagnetic chains and stripes. When the magnetization in the closing up areas of the colliding DWs is parallel (antiparallel), the walls attract (repulse), thus, they are expected to annihilate each other (to form a $2 \pi$-DW) [33], [34], [35]. The circular (helical) 
ordering in the outer shell enforces the same direction of the magnetization curling in both the colliding DWs, (it drives the sign of the DW parameter $k^{\prime \prime}$ to be the same as the sign of $d$ ). Therefore, we expect the collision to result in the annihilation of the DWs observed in [36], [37].

\section{CONCLusions}

Within a simple model of the inner core of the amorphous ferromagnetic microwire, we have established basic types of the DWs and determined the corresponding regimes of the LLG-equation parameters. The single constant of the effective DM-like anisotropy is capable to contain necessary information about the complex ordering of the outer shell that results from the permanent magnetostriction and magnetostatics. Correct evaluation of this constant allows interpretation of size and stress effects in the DW motion. The model appeared to reproduce main features of the DW shape in periodicallyconstricted nanowires. Hence, it passed a test of the validity to prototype systems which simulate the amorphous microwires, (while carrying out direct numerical simulations at the micrometer scale is an extremely challenging task).

Finally, we mention the applicability of the effective DW description to microwires coated with glassy and magnetic layers (bi-magnetic microwires) which are of recent interest with regard to sensors [38].

\section{REFERENCES}

[1] I. Betancourt, G. Hrkac, T. Schrefl, "Micromagnetic study of magnetic domain structure and magnetization reversal in amorphous wires with circular anisotropy”, J. Magn. Magn. Mat., vol. 323, no. 9, pp. 11341139, Sep. 2011;

[2] L. Stoleriou, C. Pinzaru, A. Stancu, "Micromagnetic analysis of switching and domain structure in amorphous metallic nanowires", Appl. Phys. Lett., vol. 100, no. 12, pp. 122404-1-122404-4, March 2012.

[3] J. Ye, R.P. del Real, G. Infante, M. Vazquez, "Local magnetization profile and geometry magnetization effects in microwires as determined by magneto-optical Kerr effect", J. Appl. Phys., vol. 113, no. 4, pp. 043904-1-043904-6, Jan. 2013.

[4] H. Chiriac, T.-A. Ovari, "Novel trends in the study of magnetically soft Co-based amorphous glass-coated wires", J. Magn. Magn. Mat., vol. 323, no. 23, pp. 2929-2940, Dec. 2011.

[5] M. Ipatov, V. Zhukova, J. Gonzalez, A. Zhukov, "Magnetoimpedance hysteresis in amorphous microwires induced by core-shell interaction", Appl. Phys. Lett., vol. 105, no. 12, pp. 122401-1-122401-5, Sep. 2014.

[6] H. Chiriac, T.-A. Ovari, "Amorphous glass-covered magnetic wires: preparation, properties, applications", Prog. Mat. Science, vol. 40, no. 5, pp. 333-407, 1996.

[7] M.-H. Phan, H.-X. Peng, "Giant magnetoimpedance materials: Fundamentals and applications", Prog. Mat. Science, vol. 53, no. 2, pp. 323420, Feb. 2008

[8] H. Chiriac, S. Corodeanu, M. Lostun, G. Stoian, G. Ababei, T.-A. Ovari, "Rapidly solidified amorphous nanowires", J. Appl. Phys., vol. 109, no. 6, pp. 063902-1-063902-4, March 2011.

[9] H. Chiriac, T. A. Ovari, Gh. Pop, "Internal stress distribution in glass covered amorphous magnetic wires", Phys. Rev. B, vol. 52, no. 14, pp. 10104-10113, Oct. 1995.

[10] J. Velazquez, M. Vazquez, A. P. Zhukov, "Magnetoelastic anisotropy distribution in glass-coated microwires", J. Mater. Res., vol. 11, no. 10, pp. 2499-2505, Oct. 1996.

[11] A. S. Antonov, V. T. Borisov, O. V. Borisov, A. F. Prokoshin, N. A. Usov, "Residual quenching stresses in glass-coated amorphous ferromagnetic microwires", J. Phys. D, vol. 33, no. 10, pp. 1161-1168, May 2000.

[12] H. Chiriac, T. A. Ovari, Gh. Pop, B Firuta, "Effect of glass removal on the magnetic behavior of $\mathrm{FeSiB}$ glass-covered wires", IEEE Trans. Magn., vol. 33, no. 1, pp. 782-787, Jan 1997.

[13] T. A. Ovari, S. Corodeanu, H. Chiriac, "Near-Surface Magnetic Structure and GMI Response in Amorphous Microwires", IEEE Trans. Magn., vol. 45, no. 10, pp. 4282-4285, Oct. 2009.
[14] K. Chichay, V. Zhukova, V. Rodionova, M. Ipatov, A. Talaat, J. M. Blanco, J. Gonzalez, A. Zhukov, "Tailoring of domain wall dynamics in amorphous microwires by annealing", J. Appl. Phys. vol. 113, no. 17, pp. 17A318-1-17A318-3, Mar. 2013;

[15] P. Klein, R. Varga, M. Vazquez, "Enhancing the velocity of the single domain wall by current annealing in nanocrystalline $\mathrm{FeCoMoB}$ microwires", J. Phys. D, vol. 47, no. 25, pp. 255001-1-255001-5, May 2014.

[16] M. Chandra Sekhar, H. F. Liew, I. Purnama, W. S. Lew, M. Tran, G. C. Han, "Helical domain walls in constricted cylindrical NiFe nanowires", Appl. Phys. Lett., vol. 101, no. 15, pp. 152406-1-152406-5, Oct. 2012.

[17] A. Chizhik, J. Gonzalez, P. Gawronski, K. Kulakowski, A. Zhukov, J. M. Blanco, "Torsion and tension stress induced transformation of surface magnetic structure in Co-rich amorphous microwires", J. Non-Cryst. Sol., vol. 353, no. 8-10, pp. 935-937, Apr. 2007.

[18] M. M. Bogdan, A. S. Kovalev, "Exact multisoliton solution of onedimensional Landau-Lifshitz equations for an anisotropic ferromagnet", JETP Lett., vol. 31, no. 8, pp. 424-427, Apr. 1980.

[19] A. Jimenez, R. P. del Real, M. Vazquez, "Controlling depinning and propagation of single domain-walls in magnetic microwires", Eur. Phys. J. B, vol. 86, no. 3, pp. 113-1-113-9, Mar. 2013.

[20] J. E. L. Bishop, "Shape, Energy, Eddy Current, and Relaxation Damping of Magnetic Domain Walls in Glassy Iron Wire", IEEE Trans. Magn., vol. 13, no. 5, pp. 1638-1645, May 1977.

[21] A. Janutka, "Externally driven transformations of vortex textures in flat submicrometer magnets", Phys. Rev. B, vol. 85, no. 18, pp. 184421-1184421-10, May 2012.

[22] K. Mohri, F. B. Humphrey, K. Kawashima, K. Kimura, M. Mizutani, "Large Barkhausen and Mateucci effects in $\mathrm{FeCoSiB}, \mathrm{FeCrSiB}$, and FeNiSiB amorphous wires", IEEE Trans. Magn., vol. 26, no. 5, pp. 1789-1791, Sep. 1990.

[23] L.V. Panina, M. Ipatov, V. Zhukova, A. Zhukov, "Domain wall propagation in Fe-rich amorphous microwires", Physica B, vol. 407, no. 9, pp. 1442-1445, May 2012.

[24] P. A. Ekstrom, A. Zhukov, "Spatial structure of the head-to-head propagating domain wall in glass-covered $\mathrm{FeSiB}$ microwire", J. Phys. D, vol. 43 , no. 20, 205001-1-205001-6, May 2010

[25] S. A. Gudoshnikov, Yu. B. Grebenshchikov, B. Ya. Ljubimov, P. S. Palvanov, N. A. Usov, M. Ipatov, A. Zhukov, J. Gonzalez, "Ground state magnetization distribution and characteristic width of head to head domain wall in Fe-rich amorphous microwire", Phys. Stat. Sol. A, vol. 206, no. 4, pp. 613-617, Apr. 2009

[26] A. Chizhik, J. Gonzalez, J. Yamasaki, A. Zhukov, J. M. Blanco, "Vortextype domain structure in Co-rich amorphous wires", J. Appl. Phys., vol. 95, no. 5, pp. 2933-2935, Feb. 2004.

[27] Yu. P. Ivanov, M. Vazquez, O. Chubykalo-Fesenko, "Magnetic reversal modes in cylindrical nanowires", J. Phys. D, vol. 46, no. 48, pp. 4850011-485001-11, Nov. 2013.

[28] R. C. O'Handley, 'Domain wall kinetics in soft ferromagnetic metallic glasses", J. Appl. Phys., vol. 46, no. 11, pp. 4996-5001, Nov. 1975.

[29] P. Klein, R. Varga, M. Vazquez, "Domain wall dynamics in nanocrystalline microwires", Phys. Stat. Sol. C, vol. 11, no. 5-6, pp. 1139-1143, May 2014.

[30] H. Chiriac, T.-A. Ovari, M. Tibu, "Effect of surface domain structure on wall mobility in amorphous microwires", J. Appl. Phys., vol. 105, no. 7, pp. 07A310-1-07A310-3, Feb. 2009.

[31] V. Zhukova, J. M. Blanco, M. Ipatov, A. Zhukov, "Magnetoelastic contribution in domain wall dynamics of amorphous microwires", Physica B, vol. 407, no.9, pp. 1450-1454, May 2012.

[32] H. Chiriac, T.-A. Ovari, A. Zhukov, "Magnetoelastic anisotropy of amorphous microwires", J. Magn. Magn. Mat. vol. 254-255, pp. 469471, Jan 2003.

[33] A. Janutka, "Complexes of Domain Walls in One-Dimensional Ferromagnets Near and Far from Phase Transition", Acta Phys. Pol. A, vol. 124, no. 1, pp. 23-28, Jul. 2013.

[34] A. Janutka, "Complexes of Domain Walls in Ferromagnetic Stripes", Acta Phys. Pol. A, vol. 124, no. 4, pp. 641-648, Oct. 2013.

[35] A. Janutka, "Short-Range Interactions of Domain Walls in Ferromagnetic Nanostripes", IEEE Magn. Lett., vol. 4, pp. 4000304-1-4000304-4, Aug. 2013.

[36] A. Chizhik, J. M. Blanco, M. Ipatov, V. Zhukova, J. Gonzalez, A. Zhukov, "Domain walls collision in Fe-rich and Co-rich glass covered microwires", EPJ Web of Conferences, vol. 40, pp. 17004-1-17004-3, Jan. 2013

[37] A. Zhukov, J. M. Blanco, A. Chizhik, M. Ipatov, V. Rodionova, V. Zhukova, "Manipulation of domain wall dynamics in amorphous 
microwires through domain wall collision”, J. Appl. Phys., vol. 114, no. 4, pp. 043910-1-043910-7, Jul. 2013.

[38] J. Torrejon, G. Infante, G. Badini-Confalonieri, K. R. Pirota, M. Vazquez, "Electroplated Bimagnetic Microwires: From Processing to Magnetic Properties and Sensor Devices", JOM, vol. 65, no. 7, pp. 890900, Apr. 2013. 